\title{
STRUCTURAL ANALYSIS OF IPC ZEOLITES AND RELATED MATERIALS USING POSITRON ANNIHILATION SPECTROSCOPY AND HIGH-RESOLUTION ARGON ADSORPTION
}

Jacek Jagiello $^{1}$, Michael Sterling ${ }^{1}$, Pavla Eliášová2 ${ }^{2}$, Maksym Opanasenko $^{2,3}$, Arnošt Zukal ${ }^{3}$, Russell E. Morris ${ }^{4}$, Marta Navaro, ${ }^{4}$ Alvaro Mayoral, ${ }^{5}$ Paolo Crivelli ${ }^{6}$, Robbie Warringham ${ }^{7}$, Sharon Mitchell ${ }^{7}$, Javier Pérez-Ramírez ${ }^{7}$, Jiří Čejka ${ }^{3,8}$

${ }^{1}$ Micromeritics Instrument Corporation, Norcross, GA 30093, USA

${ }^{2}$ Department of Physical and Macromolecular Chemistry, Faculty of Science, Charles University in Prague, Hlavova 2030, 12840 Prague 2, Czech Republic

${ }^{3}$ J. Heyrovský Institute of Physical Chemistry, Academy of Sciences of the Czech Republic, v.v.i., Dolejškova 3, 18223 Prague 8, Czech Republic

${ }^{4}$ University of St Andrews, School of Chemistry, Purdie Building, St Andrews KY16 9ST, Scotland

${ }^{5}$ Advanced Microscopy Laboratory (LMA), Nanoscience Institute of Aragon (INA), University of Zaragoza, Mariano Esquillor, Edificio I+D, Zaragoza 50018, Spain. ${ }^{6}$ Institute for Particle Physics, Department of Physics, ETH Zürich, Otto-Stern-Weg 5, CH 8093, Switzerland

${ }^{7}$ Institute for Chemical and Bioengineering, Department of Chemistry and Applied Biosciences, ETH Zurich, Vladimir-Prelog-Weg 1, 8093 Zurich, Switzerland

${ }^{8}$ Center of Research Excellence in Petroleum Refining and Petrochemicals, King Fahd University of Petroleum and Minerals, Dhahran 31261, Saudi Arabia

\begin{abstract}
The advanced investigation of pore networks of isoreticular zeolites and mesoporous materials related to the IPC family was performed using high-resolution argon adsorption experiments coupled with the development of a state-of-the-art non-linear density functional theory approach. The optimization of a kernel for model sorption isotherms for materials possessing the same layer structure, differing only in the interlayer connectivity (e.g. oxygen bridges, single- or double-four-ring building units, mesoscale pillars etc.) revealed remarkable differences in their porous systems. Using high-resolution adsorption data, the bimodal pore size distribution consistent with crystallographic data for IPC-6, IPC-7 and UTL samples is shown for the first time. A dynamic assessment by positron annihilation lifetime spectroscopy (PALS)
\end{abstract}


provided complementary insights, simply distinguishing the enhanced accessibility of the pore network in samples incorporating mesoscale pillars and revealing the presence of a certain fraction of micropores undetected by gas sorption. Nonetheless, subtle differences in the pore size could not be discriminated based on the widely-applied Tao-Eldrup model. The combination of both methods can be useful for the advanced characterization of microporous, mesoporous and hierarchical materials.

Keywords: IPC zeolites, positron annihilation spectroscopy, pore size distribution, highresolution adsorption, ADOR.

\section{Introduction}

Detailed analysis of the textural properties of microporous and mesoporous materials is crucial for the rational development of new and improved zeolites with advanced functionality. ${ }^{1 \text {, }}$ ${ }^{2}$ While X-ray diffraction provides precise information on the crystalline structure of molecular sieves, adsorption techniques are essential to quantify porosity and to relate porous properties to their performance in applications like catalysis and separations. ${ }^{3}$ As materials with increasingly refined pore systems continue to be developed, there is a growing demand for more sensitive techniques to discriminate subtle differences in the pore assembly. The importance of developing advanced textural analysis methods is even greater in the case of porous materials whose structures are not yet exactly defined or are amorphous ${ }^{4,5}$.

A novel family of materials where discrimination of the pore structures proves highly challenging is that of the IPC-n series of zeolites, which possess uniquely tunable micropore structures $^{6,7}$. In particular, a series of zeolites and pillared analogues can be readily prepared by transforming a UTL zeolite into its constituent layers (IPC-1P) via a chemical- and regioselective top-down approach and either separating the layers by pillaring ${ }^{8,9}$ or reassembling them into zeolite materials with specific micropore sizes. ${ }^{6,10}$ The complete sequence starting with the parent UTL zeolite to new crystalline zeolite structures is called ADOR (abbreviated from its main steps; Assembly-Disassembly-Organization-Reassembly). ${ }^{6}$ The key feature of the parent zeolite is the presence of a hydrolytically sensitive heteroatom, usually germanium, incorporated within the framework at a particular site. This enables the chemically selective removal of the units containing the dopant. Essentially, a weakness is incorporated into the material at a specific place ${ }^{11}$ followed by disassembly of the material into $2 \mathrm{D}$ layers and reassembling it into a new structure. ${ }^{12}$ Application of this strategy for UTL zeolite leads to a variety of zeolite materials with the same layer structure, but different interlayer connectivity. ${ }^{12}$ 
At present, the IPC family of UTL-derived materials includes 4 zeolites with the same chemical nature and different geometry/size of micropore apertures: IPC-4 (PCR) with $8 \times 10$ rings, IPC-2 (OKO) with $10 \times 12$ rings, IPC-9 with $7 \times 10$ rings, IPC-10 with $9 \times 12$ rings, plus the parent UTL with $12 \times 14$ rings (Fig. 1). Moreover, they can also be prepared in their combinations, i.e., the coexistence of two independent pore systems $8 \times 10$ and $10 \times 12$ in IPC-6 and $10 \times 12$ and $12 \times 14$ system in IPC-7 zeolite (Fig. 1). ${ }^{7}$ The design of these materials through the ADOR approach offers a unique opportunity to compare these isoreticular zeolites in terms of their diffusion/sorption and catalytic features. ${ }^{13}$
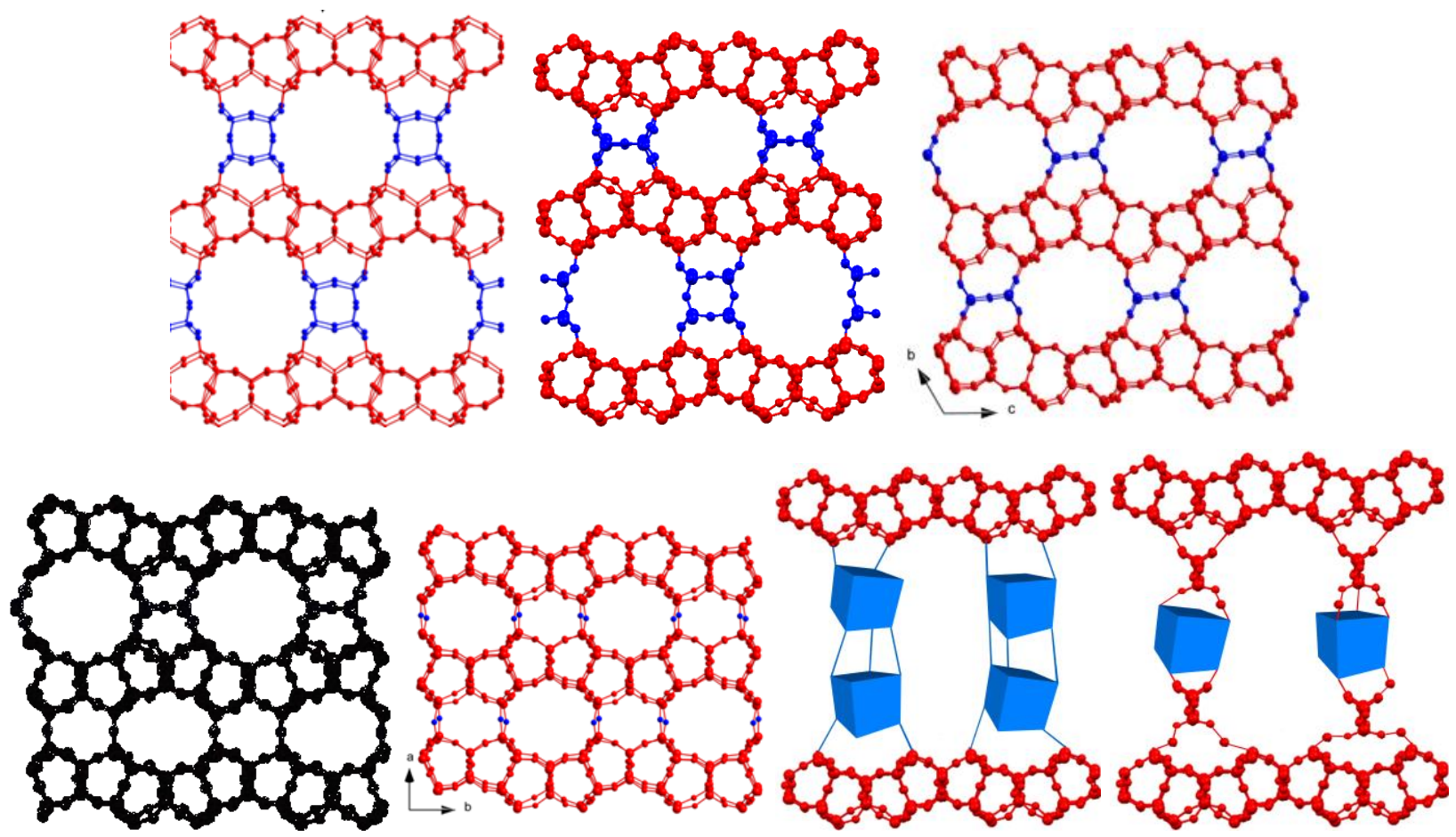

Figure 1. Structures of the UTL and IPC-1P derived materials. From left to right: (top) UTL, IPC-7, IPC-2; (bottom) IPC-6, IPC-4, IPC-1A (schematic representation), IPC-1B (schematic representation).

Various gas sorption approaches with different probe molecules have been explored to describe the porous structure of IPC zeolites and their parent UTL. ${ }^{6,14,15}$ However, none of these studies attained a satisfactory agreement between the pore size distributions (PSDs) and the crystallographic structure of the IPC materials due to the great difficulty in discriminating the subtle differences in the micropore topology. In this work, we assess the pore architecture of structurally-related zeolites and zeolite-like materials from the IPC family using argon adsorption data and the non-local density functional theory (NLDFT) model that describes the configuration of the adsorbed phase in porous materials at a molecular level. ${ }^{16}$ To get the 
maximum resolution of pore sizes we optimized the NLDFT kernel of argon adsorption isotherms used in the PSD analysis of IPC samples. By adjusting only one solid-fluid interaction parameter in the model we obtained the PSDs consistent with XRD results for all studied samples.

Another technique that has been applied for the analysis of pore networks in zeolites is that of positron annihilation spectroscopy (PALS). This technique operates on the basis that metastable ortho-positronium (o-Ps) atoms form upon the implantation of positrons into a material, which localize in voids and defects (Fig. S2 in the Supporting Information). ${ }^{10,17}$ The $o$-Ps annihilates with the lifetime related to the size of the void in which it is confined, attaining reasonable trends between the micropore size and the lifetime of $o$-Ps in different framework types (e.g. FAU, LTA, MOR). ${ }^{18-20}$ Nevertheless, the agreement is not always as satisfactory as desired, which can be attributed to a lack of understanding of the factors influencing the annihilation of $o$-Ps in zeolites. ${ }^{19}$ Apart from the analysis of $o$-Ps lifetimes, if the pore network of the material is well connected with the external surface, $o$-Ps can diffuse from the crystal and annihilate in the surrounding free volume, providing an indication of the accessibility of pores in the material. Focusing on the out-diffusion of $o$-Ps, PALS was recently demonstrated as a unique dynamic tool for probing the pore connectivity within hierarchically organized zeolites combining microand mesopores. ${ }^{21,22}$ This parameter is linked to the quality of their pore network and was shown to directly relate to the lifetime of zeolites in catalyzed reactions. ${ }^{22}$

The aim of this contribution is threefold:

1) To develop a NLDFT model (kernel) optimized for the description of microporous zeolites of the IPC family using high-resolution adsorption data,

2) To characterize these zeolites using positron annihilation lifetime spectroscopy and to compare the sensitivity of this method for distinguishing zeolites with different pore sizes,

3) To quantify the enhanced accessibility of the pore network in analogues materials having the same layer structure as the zeolites under study,

A matrix of samples including five zeolites, namely UTL, IPC-2, IPC-4, IPC-6 and IPC-7 and two pillared analogues IPC-1PI-A and IPC-1PI-B as representatives of mesoporous materials having the same structure of the layers (Fig. 1) are assessed, presenting a challenging set of crystallographically-related materials.

\section{Experimental}

\section{Synthesis}


IPC zeolites were prepared by the ADOR protocol starting from germanosilicate UTL. The synthetic procedure for UTL is detailed elsewhere. ${ }^{23}$ Briefly, a reaction mixture of molar composition $0.8 \mathrm{SiO}_{2}: 0.4 \mathrm{GeO}_{2}: 0.5 \mathrm{SDAOH}: 30 \mathrm{H}_{2} \mathrm{O}$ was prepared by dissolving germanium oxide (Aldrich) in a SDAOH (7-ethyl-6-azoniaspiro-[5.5]-undecane hydroxide) solution and addition of silica (Cab-O-Sil M5). The resulting gel was transferred into a 30-ml Teflon-lined autoclave and heated at $448 \mathrm{~K}$ for 7 days under agitation. The obtained solid product was recovered by filtration, washed with distilled water, dried overnight (363 K) and calcined in a stream of air at $823 \mathrm{~K}$ for $6 \mathrm{~h}$.

IPC-2 and IPC-4 were prepared accordingly to Ref. ${ }^{6}$ Calcined UTL was hydrolyzed in $0.1 \mathrm{M}$ $\mathrm{HCl}$ with w/w ratio of about $200 / 1$ at $90{ }^{\circ} \mathrm{C}$ overnight. The solid (IPC-1P) was isolated by filtration and centrifugation, washed with water and dried overnight. A $0.5 \mathrm{~g}$ of IPC-1P in $10 \mathrm{ml}$ of $1 \mathrm{M} \mathrm{HNO}_{3}$ solution containing $0.1 \mathrm{~g}$ of $\mathrm{Si}\left(\mathrm{CH}_{3}\right)_{2}\left(\mathrm{OCH}_{2} \mathrm{CH}_{3}\right)_{2}$ was heated at $443 \mathrm{~K}$ for $16 \mathrm{~h}$. The product (IPC-2) was isolated by centrifugation, washed and calcined at $813 \mathrm{~K}$ for $2 \mathrm{~h}$. A $0.3 \mathrm{~g}$ of IPC-1P was mixed with $20 \mathrm{~g}$ of octylamine, heated at $343 \mathrm{~K}$ for $3 \mathrm{~h}$ and then stirred at room temperature overnight. The solid (IPC-4) was isolated by centrifugation, decantation of the supernatant and drying in an open tube in air at $363 \mathrm{~K}$, and calcination at $813 \mathrm{~K}$ for $2 \mathrm{~h}$. IPC-6 and IPC-7 were synthesized by treatment of parent UTL with different aqueous solutions of $\mathrm{HCl}$ (a different concentration is required depending on the desired structure) heating at $368 \mathrm{~K}$ for 17 $\mathrm{h}$ and calcination at $823 \mathrm{~K}$ as described in Ref. ${ }^{7}$

The synthetic procedures for IPC-1A and IPC-1B mesoporous materials are described in Ref. ${ }^{24}$ IPC-1P was treated with a mixture of $40 \mathrm{wt} . \%$ tetrapropylammonium hydroxide and 25 wt. $\%$ hexadecyltrimethylammonium chloride $(\mathrm{w} / \mathrm{w}=1 / 9)$ in the ratio $1 / 50(\mathrm{w} / \mathrm{w})$. The mixture was stirred at ambient temperature overnight, the product isolated by centrifugation, washed with water, and dried at $353 \mathrm{~K}$. The obtained solid $(0.2 \mathrm{~g})$ was vigorously stirred at $333 \mathrm{~K}$ for 2 days in a chloroform solution $(5 \mathrm{ml})$ containing $0.4 \mathrm{~g}$ of octakis(tetramethylammonium)T8-siloxane (in the case of IPC-1A) or a mixture of $0.2 \mathrm{~g}$ of the same siloxane with $0.2 \mathrm{~g}$ of tetraethyl orthosilicate for (IPC-1B). The products were heated for 2 days at $368 \mathrm{~K}$, suspended in $30 \mathrm{ml}$ of $1 \mathrm{M} \mathrm{NH}_{4} \mathrm{NO}_{3}$ solution in ethanol/ $/ \mathrm{H}_{2} \mathrm{O}(\mathrm{w} / \mathrm{w}=1 / 1)$ for 2 days at room temperature, separated by centrifugation, treated with $0.2 \mathrm{M} \mathrm{HCl}$ solution in ethanol/octane mixture (w/w = 1/1) for 2 days at $333 \mathrm{~K}$, filtered off, washed with water, ethanol/octane $(\mathrm{w} / \mathrm{w}=1 / 1)$ solution, ethanol and then dried at $338 \mathrm{~K}$ overnight.

\section{Characterization}


X-ray powder diffraction measurements were carried out on a Bruker AXS D8 Advance diffractometer with a Vantec-1 detector in the Bragg-Brentano geometry using $\mathrm{CuK} \alpha$ radiation. To reduce the effect of preferential orientation of individual crystals a gentle grinding of the samples was performed before measurements.

PALS measurements were undertaken using the ETH slow positron beam. ${ }^{25}$ Powdered samples (ca. $0.1 \mathrm{~g})$ were degassed in situ under vacuum $\left(<5 \times 10^{-7} \mathrm{mbar}\right)$ at $365 \mathrm{~K}$ for $2 \mathrm{~h}$ before measurement. Monoenergetic positrons were obtained from a ${ }^{22} \mathrm{Na}$ source coupled to a rare-gas solid moderator and accelerated into the sample at $5 \mathrm{keV}$, resulting in a mean implantation depth of $500 \mathrm{~nm}$ ). Photons from annihilation events were captured by a large solid angle bismuth germanium oxide $(\mathrm{BGO})$ detector and a smaller barium fluoride $\left(\mathrm{BaF}_{2}\right)$ detector. The combined data from both sets of detectors permits increased resolution over the range of short to long annihilation lifetimes. The obtained PALS spectra were best fitted with a combination of 4 or 5 exponential contributions corresponding to the annihilation of para-positronium (125 ns), positrons ( $<1 \mathrm{~ns})$, and ortho-positronium in micropores ( $\mathrm{Ps}_{\text {micro1 }}$ and Psmicro2, 1-10 ns), in mesopores (Psmeso, 20-80 ns), and in vacuum ( $\left.\mathrm{Ps}_{\mathrm{vac}},>130 \mathrm{~ns}\right)$. Due to the time resolution of the instrument (ca. $0.9 \mathrm{~ns}$ ), the short-lived para-positronium and positron components were sometimes mixed. The relative lifetimes and intensities, collected in Table S1, were extracted using a combination of a direct deconvolution combined with Maximum Entropy regularization methods in MELT $^{26}$ and a fitting method based on Markov chain Monte-Carlo Bayesian inference in the PAScual data suite. ${ }^{27}$ For comparative purposes, the $o$-Ps intensity values were normalized by the total amount of $o$-Ps formed to eliminate variations in the absolute intensity arising due to differences in the sample quantity or composition.

The argon adsorption isotherms for UTL and the IPC samples were measured at its boiling point $(87 \mathrm{~K})$ using a high-resolution Micromeritics 3Flex instrument equipped with a highvacuum system, three micropore ports and three 0.1 Torr pressure transducers. Prior to argon adsorption, the zeolite samples were degassed at $383 \mathrm{~K}$ under turbo molecular pump vacuum until the residual pressure of $0.5 \mathrm{~Pa}$ was reached. After further heating at $383 \mathrm{~K}$ for $1 \mathrm{~h}$ the temperature was increased to $523 \mathrm{~K}$ and maintained for $8 \mathrm{~h}$.

Transmission electron microscopy analysis of the crystal size and morphology was performed on a FEI Tecnai F30 FEG instrument operated at $300 \mathrm{kV}$. The samples were directly dispersed as dry powders onto lacey carbon-coated copper grids. Powder samples for high-resolution transmission electron microscopy (HRTEM) studies were crushed with a mortar and pestle. Then they were dispersed in a few drops of ethanol onto a copper grid coated with ultra-thin holey carbon film (Ted Pella Inc.). For the zeolites IPC-2 and IPC-7 atomic resolution spherical 
aberration (Cs) corrected STEM-HAADF image acquisition was carried out on a TITAN X-FEG 60-300, operated at $300 \mathrm{kV}$ used in scanning transmission mode (STEM). The microscope is equipped with a high brightness X-FEG emission gun and a monochromator (not excited for the current experiments). The column is fitted with a CEOS spherical aberration corrector of the electron probe, an EDAX EDS detector, a Fischione HAADF detector and a Gatan BF and DF image detectors (not used). The aberration of the microscope was corrected using a gold standard sample. Beam convergence was set to $17.7 \mathrm{mrad}$ half-angle yielding to a probe size, after correction, of $0.8 \mathrm{~A}$. The total dwell time per image varied from 5 to $16 \mathrm{~s}$ depending on the magnification and beam damage observed, keeping the beam current below than $10 \mathrm{pA}$. For IPC1A and IPC-1B the microstructures were investigated using high resolution transmission electron microscopy (HRTEM) on a Jeol JEM-2011 electron microscope operating at an accelerating voltage of $200 \mathrm{kV}$. The HRTEM images were recorded using a Gatan 794 CCD camera. The camera length, sample position and magnification were calibrated using standard gold film methods.

\section{Results and discussion}

IPC zeolites prepared by the ADOR protocol are highly crystalline solids (except IPC-1A and IPC-1B) as confirmed by powder X-ray diffraction (Fig. 2). The connectivity of the silica layers (with UTL topology) varied for each zeolite, which is evidenced by the position of the dominant peak corresponding to the interlayer distance. The parent UTL zeolite has large double-four-ring (D4Rs) units between the layers giving rise to $14 \times 12$-ring channels. The interlayer peak 200 is located at $6.1^{\circ} 2 \theta$. The daughter zeolites IPC-2 (12×10-ring) and IPC-4 (10×8-ring) contain smaller connecting units, single-four-ring (S4R) and oxygen bridges, respectively. With the smaller connecting unit and thus decreased interlayer distance, the interlayer peak is shifted to higher $2 \theta$ angles. 

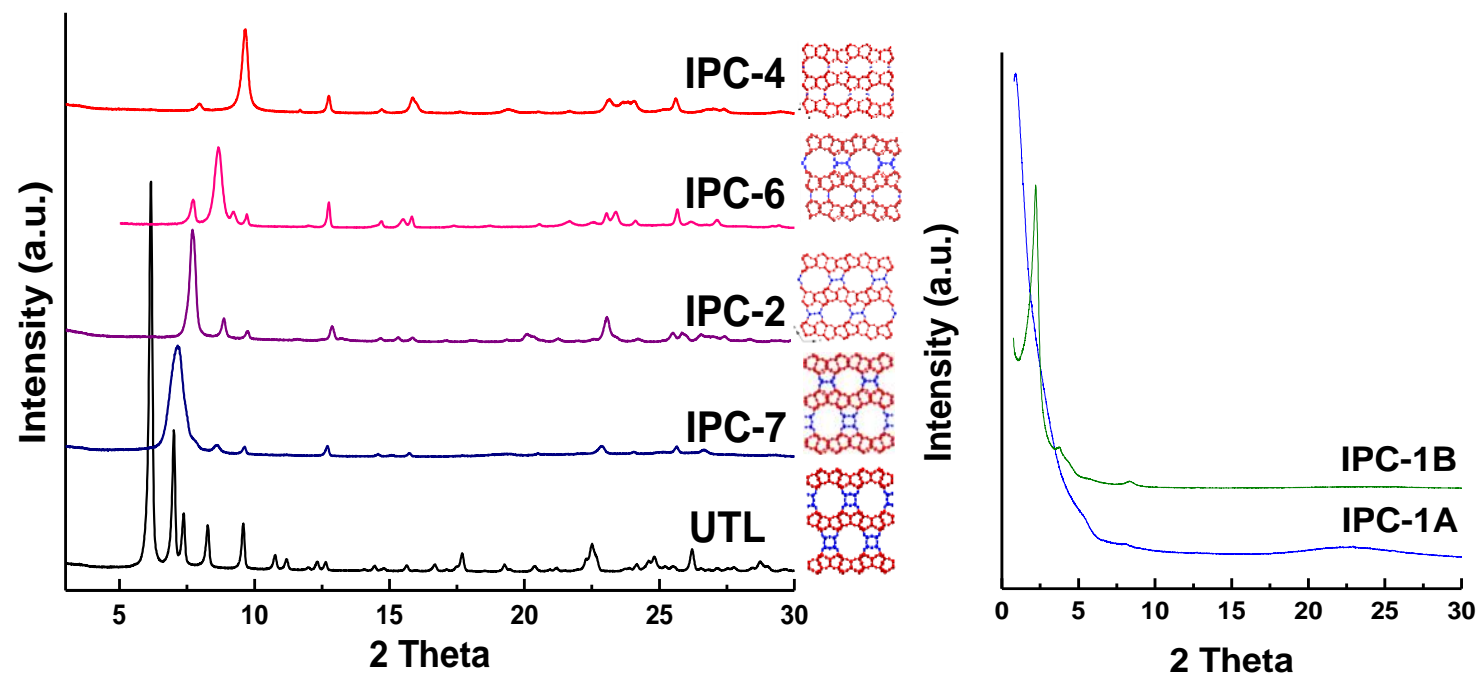

Figure 2. X-ray powder diffraction patterns of parent UTL and IPC-7, IPC-2, IPC-6 and IPC-4 zeolites (left) and mesoporous IPC-1A and IPC-1B (right).

Zeolites IPC-6 and IPC-7 combine two types of connections in a ratio close to 50:50. In IPC-7 approximately half of the layers are connected with D4R and half with S4R. On the other hand, in IPC-6 half of the layers are connected with S4R and half by oxygen bridges. Therefore, their interlayer peaks correspond to the average distance between the layers. All these zeolites are expected to be purely microporous. In contrast, in pillared IPC-1A and IPC-1B the 3D crystalline order is less pronounced (low intensity of the peaks at high $2 \theta$ ) and low angle diffraction lines dominate, which is typical for mesoporous materials. The overview of their channel diameters and textural properties are presented in Table 1. The difference in IPC-1P layers connectivity in IPC-materials can be also followed in TEM images of respective samples (Fig. 3).

Table 1 Structural and textural properties of the materials under investigation.

\begin{tabular}{|c|c|c|c|c|c|c|c|c|}
\hline \multirow{2}{*}{ Material } & \multicolumn{5}{|c|}{ Poresystem (in §̊) } & \multicolumn{2}{|c|}{ Pore volume, $\mathrm{cm}^{3} / \mathrm{g}$} & \multirow{2}{*}{$\begin{array}{c}\text { Mean } \\
\text { pore } \\
\text { width, } \AA\end{array}$} \\
\hline & Meso & 14-ring & 12-ring & 10-ring & 8-ring & Micro & $\begin{array}{l}\text { Micro } \\
+ \text { meso }\end{array}$ & \\
\hline UTL & & $9.5 \times 7.1$ & $8.5 \times 5.5$ & & & 0.246 & 0.260 & $7.38^{*}$ \\
\hline IPC-7 & & $9.5 \times 7.1$ & $\begin{array}{l}8.5 \times 5.5 \\
6.6 \times 6.2\end{array}$ & $5.4 \times 5.3$ & & 0.187 & 0.198 & $6.31^{*}$ \\
\hline IPC-2 & & & $6.6 \times 6.2$ & $5.4 \times 5.3$ & & 0.175 & 0.210 & $6.06^{*}$ \\
\hline
\end{tabular}




\begin{tabular}{|c|c|c|c|c|c|c|c|}
\hline IPC-6 & & $6.6 \times 6.2$ & $\begin{array}{l}5.4 \times 5.3 \\
5.8 \times 3.8\end{array}$ & $4.5 \times 3.6$ & 0.144 & 0.162 & $5.71^{*}$ \\
\hline IPC-4 & & & $5.8 \times 3.8$ & $4.5 \times 3.6$ & 0.120 & 0.141 & $5.31^{*}$ \\
\hline IPC-1A & 81.1 & & & & 0.015 & 0.867 & 81.1 \\
\hline IPC-1B & 28.9 & & & & 0.017 & 0.882 & 28.9 \\
\hline
\end{tabular}

Micro (w $<20 \AA$ ) and mesopore $(20<\mathrm{w}<500 \AA)$ volumes were calculated from the NLDFT analysis of the pore size distribution. (*) Mean values were calculated in the micropore range only.

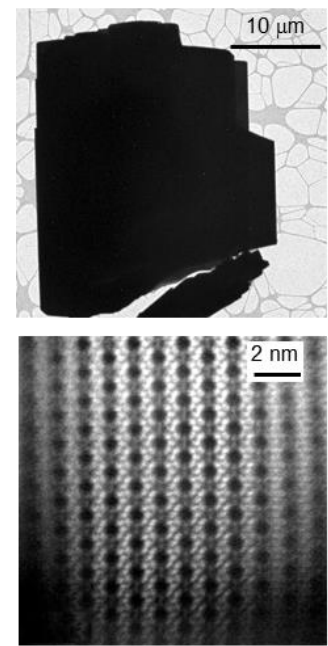

UTL
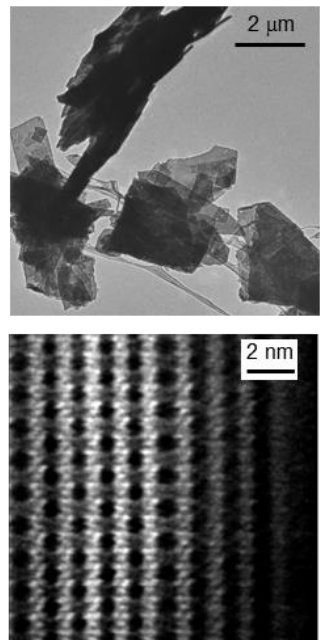

IPC-2
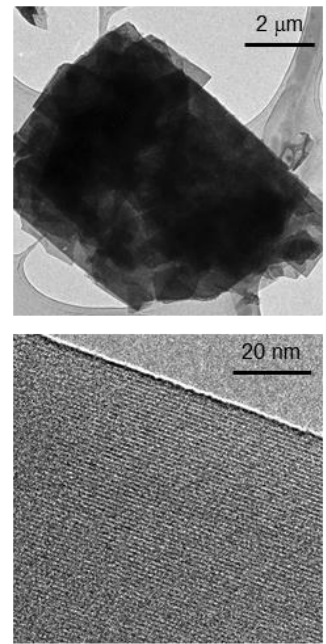

IPC-4
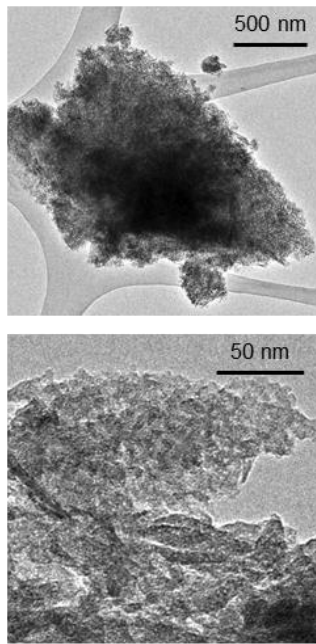

IPC-1A
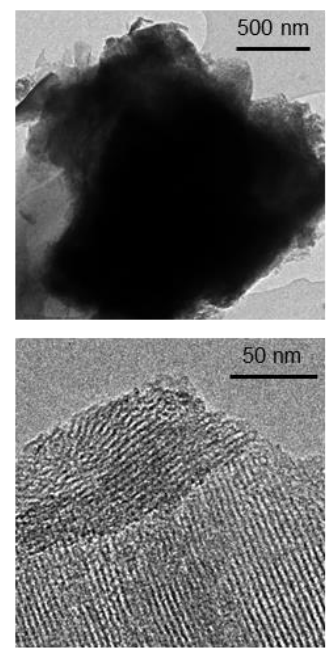

IPC-1B

Figure 3. TEM examination of selected IPC zeolites at low and high magnification. The images reveal the variation in crystal size and morphology during the ADOR process as well as the distinct mesopore topology of the pillared samples.

\section{Adsorption isotherms and pore size distribution}

Fig. 4 shows the argon isotherms of the samples measured at $87 \mathrm{~K}$. The rectangular shape of the isotherms of UTL, IPC-2, IPC-4, IPC-6 and IPC-7 are representative of zeolite structures consisting almost uniquely of micropores with a practical absence of mesopores. The small increase in adsorption at high relative pressure $\left(p / p_{\mathrm{S}}>0.95\right)$ is evidently due to argon condensation in interstitial voids among the zeolite particles, which is most pronounced for UTL. The initial vertical parts of the isotherms are so similar that they virtually overlap in linear coordinates. More detailed comparison of the isotherms in logarithmic coordinates in Fig. 5 reveals the characteristic sigmoidal shape, typical for ordered microporous materials. Here it is 
clearly seen how small diameter pores (8×10-ring) in IPC-4 and IPC-6 are filled first at very low relative pressures followed by the larger 10×12-ring in IPC-2 and IPC-7 and finally the extra-large pores $12 \times 14$-ring in UTL. The zeolites that partially share the same channel system, IPC-4 and IPC-6 with $8 \times 10$-ring, and IPC-2 and IPC-7 with $10 \times 12$-ring, have a similar profile of isotherms in semi-logarithmic coordinates (Fig. 5). Their common smaller channels are filled first starting at the same relative pressures. And they differ in higher relative pressures due to the presence of the second system of larger independent channels. IPC-1A and IPC-1B derivatives possess a negligible amount of micropores (Table 1). Adsorption isotherms for both these materials were characterized by quite narrow mesopore size distribution (Fig. 5). The average mesopore diameter depends on the method of preparation and totaled about 81 and $29 \AA$ for IPC$1 \mathrm{~A}$ and IPC-1B, respectively.
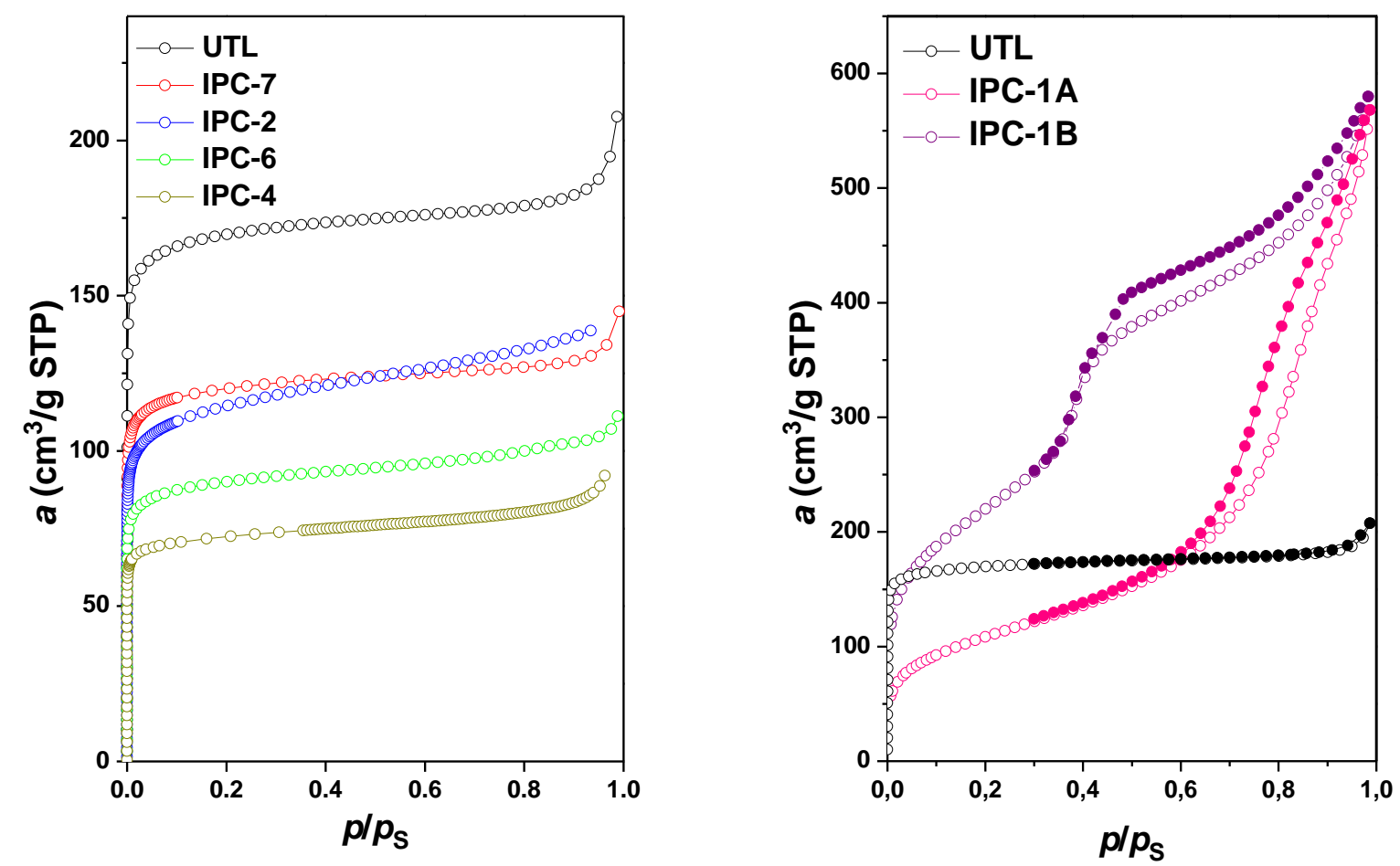

Figure 4. Argon adsorption isotherms (in linear coordinates) measured at $87 \mathrm{~K}$ on the materials under investigation. 


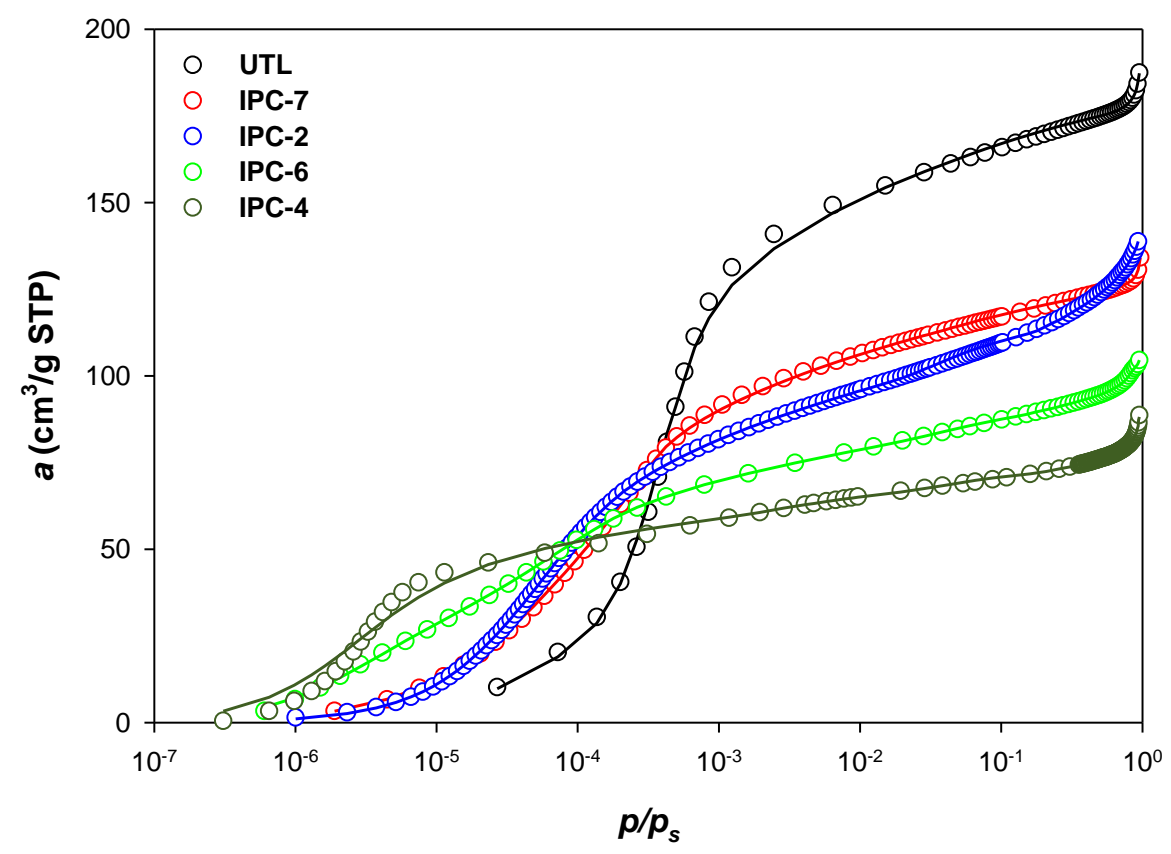

Figure 5. Argon adsorption isotherms (in semi-logarithmic coordinates) of the zeolites studied (87 K). Solid lines represent fits of the cylindrical NLDFT model to the experimental data.

The method for extracting the pore size distribution (PSD) from the adsorption isotherm is based on the assumption that the experimentally measured isotherm can be expressed in terms of the adsorption integral equation first proposed by Seaton et al. ${ }^{28}$ for the PSD analysis of porous carbons. Detailed description of PSD determination procedure can be found in Supporting Information. As a result of this procedure we obtain the fit to the isotherm data and the PSD of a sample. The results of the PSD analysis of the adsorption isotherms of UTL and IPC zeolites using our simple cylindrical NLDFT model are shown in Fig. 5 and 6 and in Table 1. A quantitatively good fit of the model to the data is obtained for all samples (Fig. 5) except for IPC-4 sample where small deviations are observed in the low pressure range. The structural parameters of the samples listed from top to bottom for UTL, IPC-7, IPC-2, IPC-6 and IPC-4 (in Table 1) show decreasing trend in their values of the micropore volume and the mean pore width calculated as the first-order moment of the PSD. 

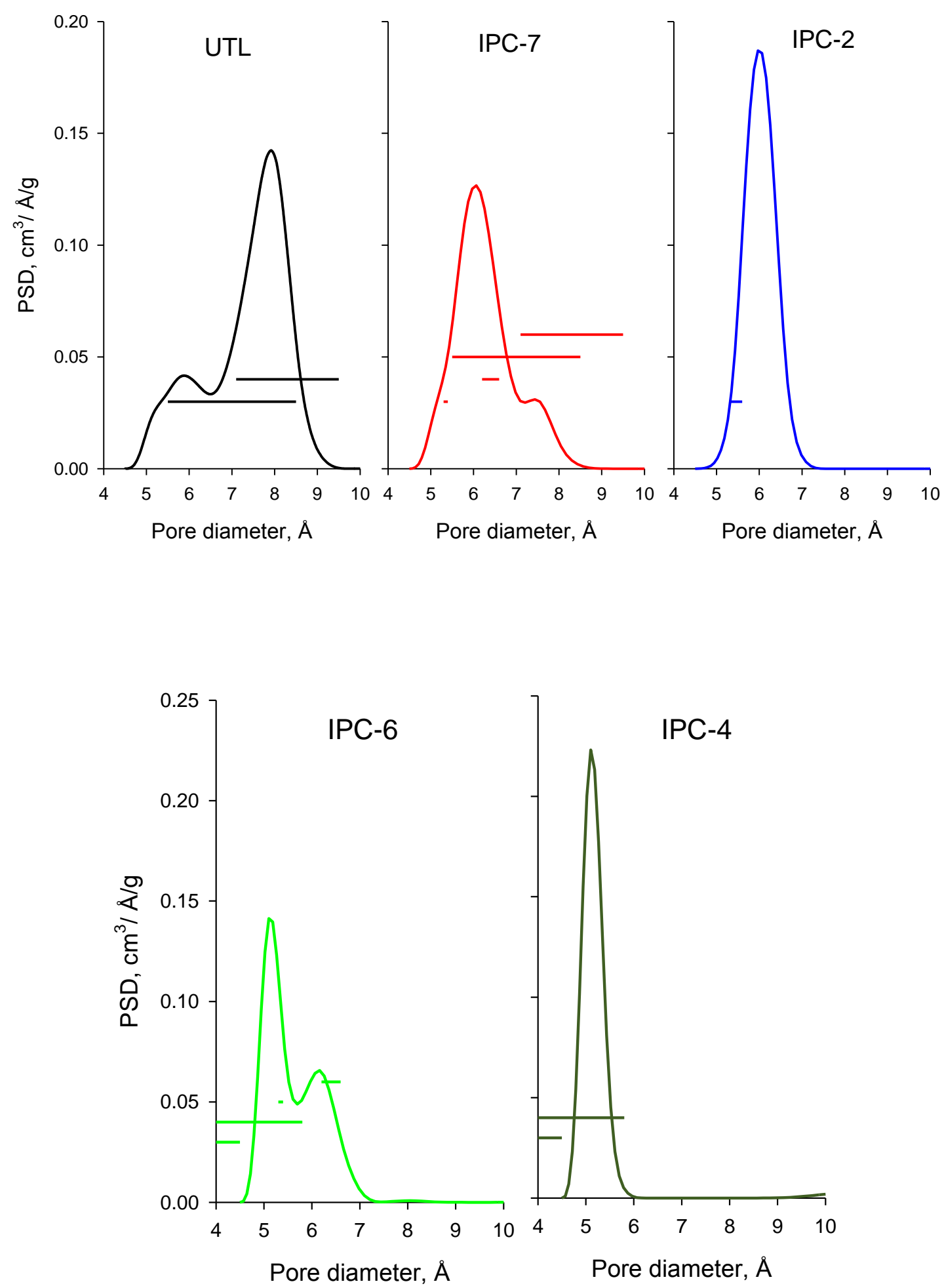

Figure 6. Pore size distributions of the samples calculated from the argon isotherms. Horizontal bars represent the variation of the diameter values of each channel of the sample.

In the discussion of the evaluated PSDs (Fig. 6), it is important to keep in mind that the ideal cylindrical model used in the analysis is only a simplified representation of the actual/real pore structure. The main simplifications of the real pore structure in UTL and IPC zeolites are that the 
model neglects the fact that there are intersections between the orthogonal channels in these materials and assumes that the channels are perfect cylinders with circular cross sections. In a more realistic description based on the structural analysis (from X-ray powder diffraction data) these channels are irregular, intercrossing (2-dimensional) and each of them can be characterized by two different widths (diameters) measured in different directions. To address this feature in the PSD graphs (Fig. 6) we included horizontal bars describing irregularity of the channels. Each bar represents the range given by two diameter values for each channel of the sample (Table 1). For example, UTL has two channels each characterized by diameters of $7.1-9.5 \AA$ and $5.5-8.5$ $\AA$. In this case the ranges of pore diameters describing both channels partially overlap and practically cover the same range as the calculated PSD that shows two peaks corresponding to the two channels of this sample (Fig. 6). This demonstrates the consistency between the calculated PSD as the effective combination of the PSDs related to the individual channels and the structural analysis data represented graphically by the horizontal bars. Similar arguments can be applied to all calculated PSDs, which are more or less consistent with the XRD results in terms of covering the same pore size range. Partial resolution of the PSD peaks related to different channels can be seen only for UTL, IPC-6 and IPC-7.

In the case of IPC-2 and IPC-4 samples the range of the overlapping peaks related to the individual channels is so narrow that the peaks cannot be resolved and only one common peak is observed. It is important to realize at this point that our method has limitations with respect to resolution and the choice of the conservative optimal solution is probably the best we can do in this case.

\section{Dynamic assessment of the pore network by PALS}

Deconvolution of the PALS spectra (Table S2) acquired for the purely microporous UTL, IPC-2 and IPC-4 samples identified three $o$-Ps lifetime components (Fig. 7): two below $10 \mathrm{~ns}$ attributed to annihilation within micropore-sized volumes (Psmicro1 and $\mathrm{Ps}_{\text {micro2 }}$ ) and a single component corresponding to annihilation in vacuum (close to $142 \mathrm{~ns}, \mathrm{Ps}_{\mathrm{vac}}$ ). ${ }^{21}$ Since $o$-Ps are sufficiently long-lived to diffuse through the pore network, the relative intensity of each lifetime component carries information not only about the amount, size, but also about the connectivity of micro- and mesopores within the samples. In particular, due to the quantum mechanical nature of the diffusion within pores of this size range, $o$-Ps will preferentially migrate to accessible pores of increasing size and if possible to vacuum driven by the decreasing zero-point energy. The relative contribution of the two micropore components of the UTL, IPC-2, and IPC-4 zeolites of close to $90 \%$ is fully consistent with the plate-like morphology of these materials. 
Since the channels run parallel to the layers, $o$-Ps must diffuse over the full length of the plate (in the order of $\mu \mathrm{m}$ ) to escape the crystal, increasing the likelihood of decay in the micropores and resulting in a low probability of annihilation in vacuum as o-Ps will likely decay in the micropores. Although the daughter (IPC-2, IPC-4) zeolites exhibit a decreased crystal size with respect to the UTL parent (Fig. 3), which could increase the probability of out diffusion, this effect will be compensated by the reduced characteristic diffusion length of $o$-Ps owing to the smaller micropore dimensions.

Derivation of pore sizes from positronium annihilation lifetimes is widely approached by application of the extended Tao-Eldrup model. ${ }^{29}$ Here, the two $o$-Ps components observed below $10 \mathrm{~ns}$ correspond to sizes of $6.2,5.4$ or $6.0 \AA$ (Psmicro1) and 10.4, 9.0, or $9.7 \AA$ (Psmicro2) assuming a square channel geometry for UTL, IPC-2 and IPC-4, respectively. The observation of these distinct lifetimes indicates that $o$-Ps atoms experience at least two dissimilar micropore environments within the samples, although the first contribution clearly dominates in all cases. While slightly higher lifetimes are evidenced for the UTL zeolite, consistent with the larger channel sizes of this structure, the lifetimes of the micropore components in IPC-2 and IPC-4 do not exhibit an obvious relation with the size of the connecting units. It should be noted that the Tao-Eldrup model is limited to pores of square channel and discrete spherical geometries, and does not account for pore interconnectivity or for the fact that due to the energetic driving forces $o$-Ps may not equally sample all of the pores within a material. Thus, it is not surprising that the estimated pore sizes do not match the crystallographic pore sizes. Although the presence of framework defects is not likely as ${ }^{29}$ Si MAS NMR spectra and XRD confirmed the high quality of this material, ${ }^{6}$ the second micropore component could indicate the presence of a certain fraction of stacking faults or other small defects in the samples not detectable by these methods.

Analysis of the pillared IPC-1A and 1B samples evidenced an additional component between 60-80 ns, attributed to $o$-Ps annihilation in mesopore-sized volumes. The low intensity of this component (5-10\%) coupled with the high vacuum contribution (ca. 50\%) suggests the number of annihilation events in these mesopore-sized volumes are relatively low. This indicates that the mesopore domains are well-connected with the external surface, facilitating the escape of $o$-Ps. No clear relation is attained between the average mesopore sizes estimated by NLDFT (29 and $81 \AA$, respectively) and by the Tao-Eldrup model, which indicates similar values of ca. 57 and $52 \AA$ (for a square channel). Consistent with the results of Ar adsorption, comparison of the TEM images reveals significant differences in the mesopore topology (Fig. 3). While both samples appear highly mesoporous, IPC-1A presents larger mesopores which appear less ordered than the uniform channel-like structures evidenced in IPC-1B. The distinct mesopore 
architecture together with the significant extent of out diffusion from the crystal could explain the lack of a good agreement in the derived mesopore sizes, again reflecting the inadequacy of current models to relate the PALS response to the pore structure. Interestingly, although the contribution in the micropores (ca. 40\%) is less than half that of the purely microporous materials, it remains significant and cannot be explained based on the negligible values derived by adsorption data $\left(\mathrm{V}_{\text {micro }}=0.015-0.017 \mathrm{~cm}^{3} / \mathrm{g}\right)$. Structurally, IPC-1A and 1B incorporate cubic $(\mathrm{O}-\mathrm{Si}-\mathrm{O})_{8}$ linkers separating the layers by ca. 8 and $3 \mathrm{~nm}$, respectively, and thus no significant microporosity is expected. Such an observation could be ascribed to the incomplete hydrolysis of the UTL layers. However, a noticeably reduced lifetime is observed for $\mathrm{Ps}_{\text {microl }}$ in the pillared samples with respect to UTL, and the presence of non-hydrolyzed UTL layers is not detected by powder XRD. An alternative possibility could be that some of the hydrolyzed layers have randomly condensed without incorporating the pillaring species. Since the extent of micropore voids should be small, the fact that the components remain non-negligible strongly suggests that they are not interconnected with the mesopore network. As o-Ps atoms forms in situ upon positron implantation, they can probe isolated microporous regions that may not be accessible by gas sorption providing a measure of the structural uniformity. Full details of the data presented in Fig. 7 are collected in Table S2 in the Supporting Information.

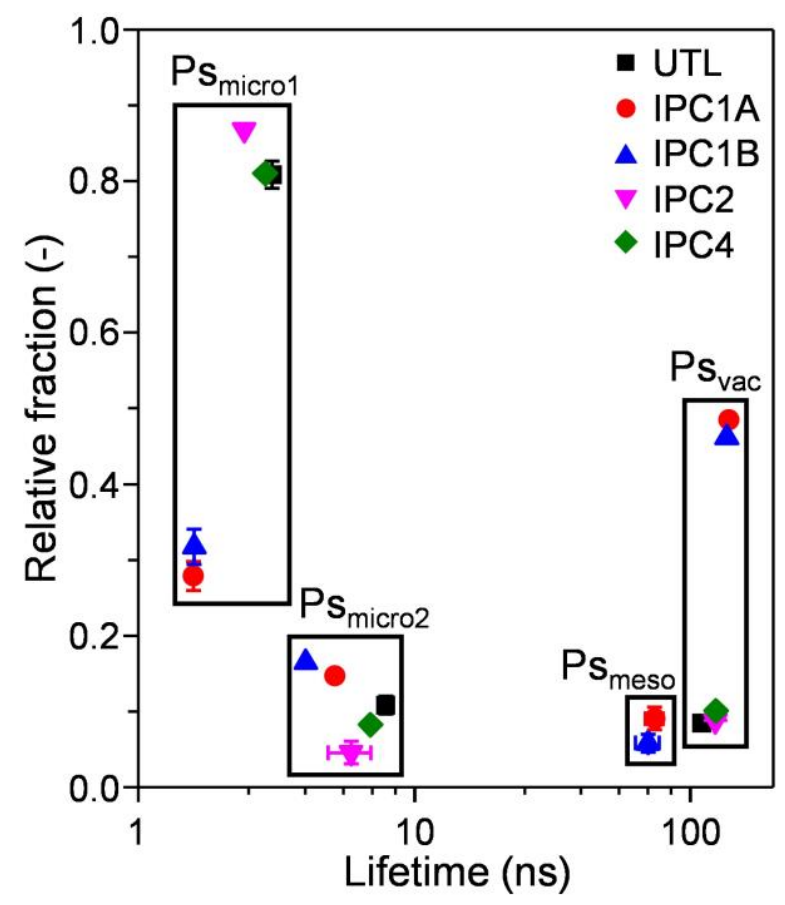

Figure 7. The normalized $o$-Ps values from the measured samples, indicating the fractions annihilating in the micropore, mesopore and vacuum, respectively.

\section{Conclusions}


We have assessed the potential of a combination of high-resolution argon adsorption and positron annihilation lifetime spectroscopy to characterize the textural properties of a series of novel isoreticular zeolites and related materials having the same (IPC-1P) structure of their layers.

The analysis of the UTL-derived materials has shown that PALS is sensitive to the structural differences between zeolites from the IPC family. In particular, the dynamic assessment by this technique could easily differentiate between the different accessibility of the pore network in the purely microporous UTL, IPC-2 and IPC-4 samples and the predominantly mesoporous pillared structures of IPC-1A/B. On the basis of the current measurements, it was not possible to relate the lifetime of ortho-positronium in the micropores of the UTL, IPC-2 and IPC-4 samples to the small differences in the crystallographic structure. In this regard, the development of a fundamental framework to describe the diffusion of the ortho-positronium in zeolites will be imperative to obtain improved insight. Capping of the external surface to isolate the ortho-positronium within the zeolitic domains could also facilitate the more accurate determination of the micropore lifetime. Analysis of the pillared IPC-1A and IPC-1B zeolites also revealed a non-negligible fraction of ortho-positronium annihilating within micropores, which is ascribed to the presence of residual microporous regions that were undetectable by argon adsorption.

Pore size distributions calculated from high-resolution argon isotherms using an adsorbent with cylindrical pores as kernel represent simple models of the porous structure of UTL and structurally related IPC zeolites. The remarkable feature of this model, which is composed from cylindrical pores of a given distribution, is that the model provides the same argon isotherm as experimental one recorded on the investigated sample. This model provides a basis for an analysis of different processes taking place inside the pores and depending on their size, which are principally consistent with structural data.

\section{Acknowledgements}

Dr. Asier Zubiaga is acknowledged for discussions on the PALS analysis. ETH authors thanks for the grant ETH 33 15-1. PE and JČ acknowledge the financial support from the Czech Science Foundation (P106/12/0189).

\section{$\underline{\text { References }}$}


1. J. C. Groen, L. A. A. Peffer and J. Perez-Ramirez, Micropor. Mesopor. Mater., 2003, 60, 1-17.

2. A. Stein, Adv. Mater., 2003, 15, 763-775.

3. A. Corma, Chem. Rev., 1997, 97, 2373-2419.

4. S. Mitchell, A. B. Pinar, J. Kenvin, P. Crivelli, J. Kärger and J. Pérez-Ramírez, Nat. Commun., 2015, 6, 8633.

5. Y. Wei, T. E. Parmentier, K. P. de Jong and J. Zečević, Chem. Soc. Rev., 44, 7234-7261.

6. W. J. Roth, P. Nachtigall, R. E. Morris, P. S. Wheatley, V. R. Seymour, S. E. Ashbrook, P. Chlubna, L. Grajciar, M. Polozij, A. Zukal, O. Shvets and J. Čejka, Nat. Chem., 2013, 5, 628-633.

7. P. S. Wheatley, P. Chlubná-Eliášová, H. Greer, W. Zhou, V. R. Seymour, D. M. Dawson, S. E. Ashbrook, A. B. Pinar, L. B. McCusker, M. Opanasenko, J. Čejka and R. E. Morris, Angew. Chem. Int. Ed., 2014, 53, 13210-13214.

8. M. Shamzhy, M. Mazur, M. Opanasenko, W. J. Roth and J. Čejka, Dalton Trans., 2014, 43, 1054810557

9. M. Opanasenko, M. Shamzhy, F. Yu, W. Zhou, R. E. Morris and J. Cejka, Chem. Sci., 2016, 10.1039/C5SC04602E.

10. W. Schmidt, in Handbook of Porous Solids, Wiley-VCH Verlag GmbH, 2008, pp. 506-532.

11. R. E. Morris and J. Čejka, Nat. Chem., 2015, 7, 381-388.

12. P. Eliášová, M. Opanasenko, P. S. Wheatley, M. Shamzhy, M. Mazur, P. Nachtigall, W. J. Roth, R. E. Morris and J. Čejka, Chem. Soc. Rev., 2015, 44, 7177-7206.

13. N. Žilková, P. Eliášová, S. Al-Khattaf, R. E. Morris, M. Mazur and J. Čejka, Catal. Today, DOI: 10.1016/j.cattod.2015.09.033.

14. M. V. Shamzhy, O. V. Shvets, M. V. Opanasenko, P. S. Yaremov, L. G. Sarkisyan, P. Chlubna, A. Zukal, V. R. Marthala, M. Hartmann and J. Čejka, J. Mater. Chem., 2012, 22, 15793-15803.

15. M. Mazur, P. S. Wheatley, M. Navarro, W. J. Roth, M. Položij, A. Mayoral, P. Eliášová, P. Nachtigall, J. Čejka and R. E. Morris, Nat. Chem., 2016, 8, 58-62.

16. K. S. W. Sing, F. Rouquerol and J. Rouquerol, in Adsorption by Powders and Porous Solids (Second Edition), Academic Press, Oxford, 2014, pp. 159-189.

17. D. W. Gidley, H.-G. Peng and R. S. Vallery, Annu. Rev. Mater. Res., 2006, 36, 47-79.

18. H. Nakanishi and Y. Ujihira, J. Phys. Chem., 1982, 86, 4446-4450.

19. Y. Ito, T. Takano and M. Hasegawa, Appl. Phys. A, 1988, 45, 193-201.

20. M. Debowska, J. C. Abbe and G. Duplatre, Phys. Status Solidi B, 1988, 146, 91-96.

21. M. Milina, S. Mitchell, P. Crivelli, D. Cooke and J. Perez-Ramirez, Nat. Commun., 2014, 5.

22. M. Milina, S. Mitchell, D. Cooke, P. Crivelli and J. Perez-Ramirez, Angew. Chem. Int. Ed., 2015, 54, 1591-1594.

23. O. V. Shvets, N. Kasian, A. Zukal, J. Pinkas and J. Čejka, Chem. Mater., 2010, 22, 3482-3495.

24. M. Opanasenko, W. O. N. Parker, M. Shamzhy, E. Montanari, M. Bellettato, M. Mazur, R. Millini and J. Čejka, J. Am. Chem. Soc., 2014, 136, 2511-2519.

25. P. Crivelli, U. Gendotti, A. Rubbia, L. Liszkay, P. Perez and C. Corbel, Phys. Rev. A, 2010, 81.

26. A. Shukla, M. Peter and L. Hoffmann, Nucl. Instrum. Methods A, 1993, 335, 310-317.

27. C. Pascual-Izarra, A. W. Dong, S. J. Pas, A. J. Hill, B. J. Boyd and C. J. Drummond, Nucl. Instr. Meth. Phys. A, 2009, 603, 456-466.

28. N. Seaton and J. Walton, Carbon, 1989, 27, 853-861.

29. D. W. Gidley, W. E. Frieze, T. L. Dull, A. F. Yee, E. T. Ryan and H.-M. Ho, Phys. Rev. B, 2001, 60, R5157. 